\title{
SCIMITAR SYNDROME: TWENTY YEARS' EXPERIENCE AND RESULTS OF REPAIR
}

Hani K. Najm, MD

William G. Williams, MD

John G. Coles, MD

Ivan M. Rebeyka, MD

Robert M. Freedom, MD
Background: Thirty-two patients with scimitar syndrome were seen in the period between 1975 and 1995. There were 11 male and 21 female patients. Median age at diagnosis was 7 months (mean 7.7 years, range 1 day to 70 years). Patients in whom the diagnosis was made during the first year of life (infantile group, $n=19$ ) had more severe symptoms and had a higher incidence of heart failure (11/19 vs 0/13) and of pulmonary hypertension (11/19 vs $1 / 13$ ) than did the patients in whom the diagnosis was made after age 1 year (adult group, $n=13$ ). In 17 patients the anomalous pulmonary venous drainage was repaired by baffling the vein to the left atrium. The median age at this operation was 5.8 years (mean 14.8 years, range 6 months to 70 years). Results: No deaths occurred in this surgical group during a mean follow-up period of 8.9 years (range 1.6 to 17 years). Eight patients (47\%), however, had evidence of pulmonary venous stenosis after repair, and two required reoperation for pulmonary venous obstruction. All six children in the infantile group had postoperative pulmonary venous stenosis, compared with two of 11 older patients. Postoperative quantitative pulmonary perfusion scans performed in 15 patients demonstrated reduced flow to the right lung (24\%, range $0 \%$ to 59\%). Conclusion: We conclude that age at detection of scimitar syndrome is important in predicting outcome. Surgical repair seldom results in normal blood flow to the right lung but abolishes left-to-right shunt. Postoperative pulmonary venous obstruction is prevalent, especially in the infants. ( $J$ Thorac Cardiovasc Surg 1996;112:1161-9)
$\mathrm{S}_{\mathrm{i}}^{\mathrm{in}}$ cimitar syndrome is a rare congenital anomaly. This syndrome is characterized by partial or complete anomalous pulmonary venous drainage of the right lung to the inferior vena cava (IVC). The first reported case was published in 1836 by Cooper. $^{1}$ Neill and associates ${ }^{2}$ in 1960 described the scimitar sign and offered a detailed description of the syndrome. Associated anomalies are hypoplastic

From the Divisions of Cardiovascular Surgery and Cardiology, Department of Pediatrics and Surgery, Hospital for Sick Children, and University of Toronto Faculty of Medicine, Toronto, Ontario, Canada.

Read at the Seventy-sixth Annual Meeting of The American Association for Thoracic Surgery, San Diego, Calif., April 28-May 1, 1996.

Received for publication May 6, 1996; revisions requested June 6, 1996; revisions received June 21, 1996; accepted for publication June 24, 1996.

Address for reprints: William G. Williams, MD, Chief, Cardiovascular Surgery, Division of Cardiovascular Surgery, The Hospital for Sick Children, 555 University Ave., Toronto, Ontario M5G 1X8, Canada.

Copyright (C) 1996 by Mosby-Year Book, Inc.

$0022-5223 / 96 \$ 5.00+0 \quad \mathbf{1 2 / 6 / 7 6 0 4 4}$ right pulmonary artery and lung, occasionally with abnormal bronchial anatomy, dextrocardia, and systemic arterial supply to the lower lobe of the right lung. Successful surgical repair of the syndrome was first reported by Kirklin, Ellis, and Wood ${ }^{3}$ in 1956. Scimitar syndrome forms only $3 \%$ to $5 \%$ of all cases of partial anomalous pulmonary venous drainage. ${ }^{4,5}$

Dupuis and associates ${ }^{6,7}$ identified two separate types of scimitar syndrome, the infantile and the adult forms. The infantile form is a severe form of the disease with major associated cardiac lesions, including pulmonary vascular disease, and the results of treatment are poor. This report is a review of our experience in treating patients with the scimitar syndrome and the long-term results of surgical repair.

\section{Method}

Between January 1975 and January 1995, 32 patients with a diagnosis of scimitar syndrome were seen in the Hospital for Sick Children or the Toronto Congenital Cardiac Center for Adults, Toronto, Canada. Hospital, clinic, and office records were reviewed to document clinical presentation and course of illness. All preoperative and postoperative angiograms were reviewed, and hemodynamic parameters were recorded. Pulmonary ve- 


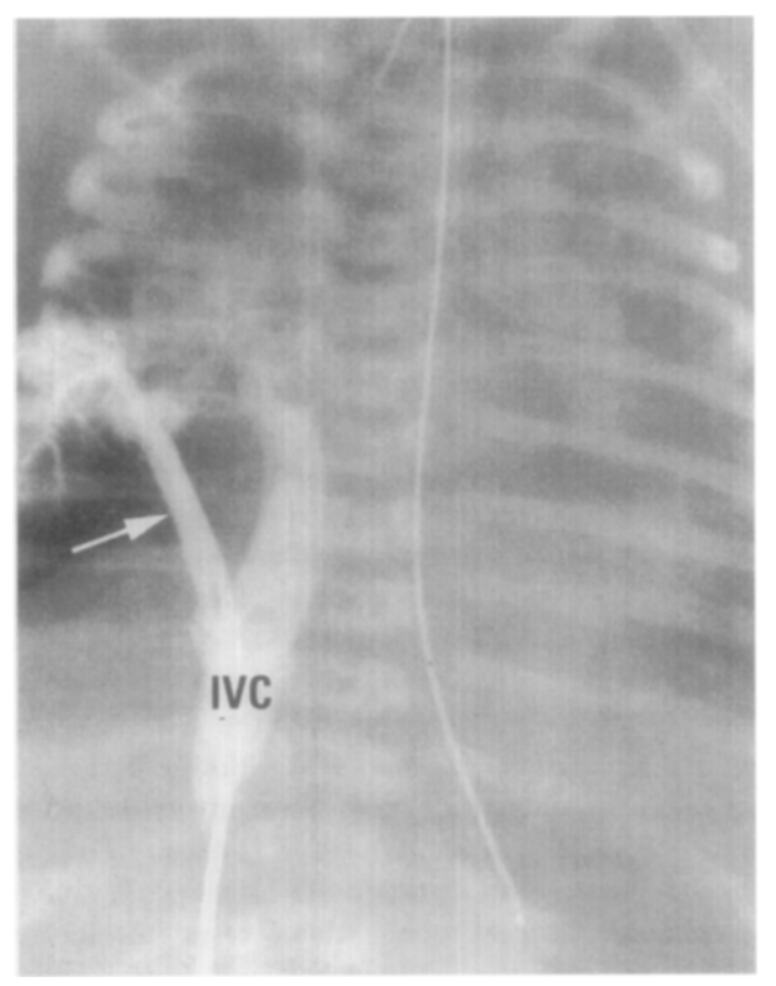

Fig. 1. Anomalous vein from the right lung draining to the IVC.

nous obstruction is defined as an angiographic stenosis or diffuse hypoplasia of the venous drainage. Quantitative pulmonary perfusion scans were available from 20 patients, 15 after repair and five without repair.

Patient characteristics. The series comprised $21 \mathrm{fe}-$ male and 11 male patients. Median age at diagnosis was 7 months (mean 7.7 years, range 1 day to 70 years). The right lung was involved in all patients. Drainage of the anomalous vein was to the IVC-atrial junction in 28 (Fig. 1), to the right atrium close to the IVC in two, and to the infradiaphragmatic IVC in two. Three patients had drainage to the IVC and to the left atrium. Associated anomalies were present in all but three patients. Table I lists the anomalies, the most common of which was an atrial septal defect. Associated defects were more frequent in the infantile group than in the adult group. Hypoplasia of the right pulmonary artery or right lung was present in 17 patients (Fig. 2, $A$ ), all of whom had systemic arterial supply, that is, sequestration (Fig. 2, B). Pulmonary vein stenosis was present on initial assessment in seven patients. Noncardiac anomalies were VATER syndrome in one patient (vertebral defects, imperforate anus, tracheoesophageal fistula, and radial and renal dysplasia) and Turner's syndrome in another.

Preoperative hemodynamics. Diagnosis was confirmed by cardiac catheterization in all but three patients, in whom echocardiography alone was used. Left-to-right shunting may occur through the right lung via the anomalous vein, or
Table I. Associated anomalies in all patients

\begin{tabular}{lccc}
\hline \multicolumn{1}{c}{ Associated anomaly } & $\begin{array}{c}\text { Infant } \\
(n=19)\end{array}$ & $\begin{array}{c}\text { Adult } \\
(n=13)\end{array}$ & $\begin{array}{c}\text { Total } \\
(n=32)\end{array}$ \\
\hline Atrial septal defect & 15 & 6 & 21 \\
Sequestration & 13 & 4 & 17 \\
Hypoplastic lung & 13 & 4 & 17 \\
Dextrocardia & 11 & 4 & 15 \\
Pulmonary vein stenosis & 5 & 2 & 7 \\
Left SVC & 2 & 2 & 4 \\
MV prolapse & 1 & 3 & 4 \\
Coarctation & 4 & 0 & 4 \\
PDA & 4 & 0 & 4 \\
Horseshoe lung & 2 & 1 & 3 \\
Azygos continuation of IVC & 1 & 2 & 3 \\
Small left ventricle & 3 & 0 & 3 \\
VSD & 2 & 0 & 2 \\
Bicuspid aortic valve & 0 & 2 & 2 \\
RVMB & 1 & 0 & 1 \\
Right aortic arch & 1 & 0 & 1 \\
Total & 78 & 30 & 108 \\
\hline
\end{tabular}

$\overline{S V C}$, Superior vena cava; $M V$, mitral valve; $P D A$, patent ductus arteriosus; $I V C$, inferior vena cava; $V S D$, ventricular septal defect; $R V M B$, right ventricular muscle band.

through an atrial defect, or from the anomalous arterial supply to the right lung. At catheterization, the calculated shunt fraction (Qp/Qs) was 2.4:1, but varied considerably from 0.9 to $5.9: 1$ (Table II). Only 15 patients had a Qp/Qs greater than $2: 1$. The mean oxygen saturation in the IVC was $83 \%$ versus $64 \%$ in the superior vena cava. The presence of an atrial septal defect in 21 patients and the presence of an arterial supply from the aorta to the right lower lobe of the lung in 17 patients complicated the estimate of left-to-right shunting through the anomalous pulmonary vein. Congenital pulmonary venous stenosis was demonstrated in seven patients. Angiography of the descending aorta demonstrated systemic arterial supply to the right lung in 17 patients.

The study group is considered in two groups depending on time of detection of scimitar syndrome, as previously recommended by Dupuis and associates. ${ }^{6,7}$ The infantile group are those children in whom the syndrome was detected in the first year of life, and the adult group is defined as those patients in whom it was detected after the first year of life.

Infantile group. The infantile group comprised 19 patients. The median age at presentation was 25 days (mean 70 days, range 1 to 335 days). There were five boys and 14 girls. All had symptoms (Table III). Symptoms included cardiac failure in 11, dyspnea in three, chest infection in two, failure to thrive in one, cyanosis in one, and VATER syndrome in one child. Pulmonary hypertension was documented in 11.

Six patients from the infantile group underwent a total of 12 palliative procedures, including occlusion of arterial collaterals in five (by catheter in four and by ligation in one) (Table IV) and coarctation repair, which was performed in one patient at 1 month of age followed by pneumonectomy at 3 months of age. Other interventions done concomitantly with arterial occlusion included ligation of patent ductus $(n=1)$, dilatation of right pulmonary vein $(n=1)$, dilation 

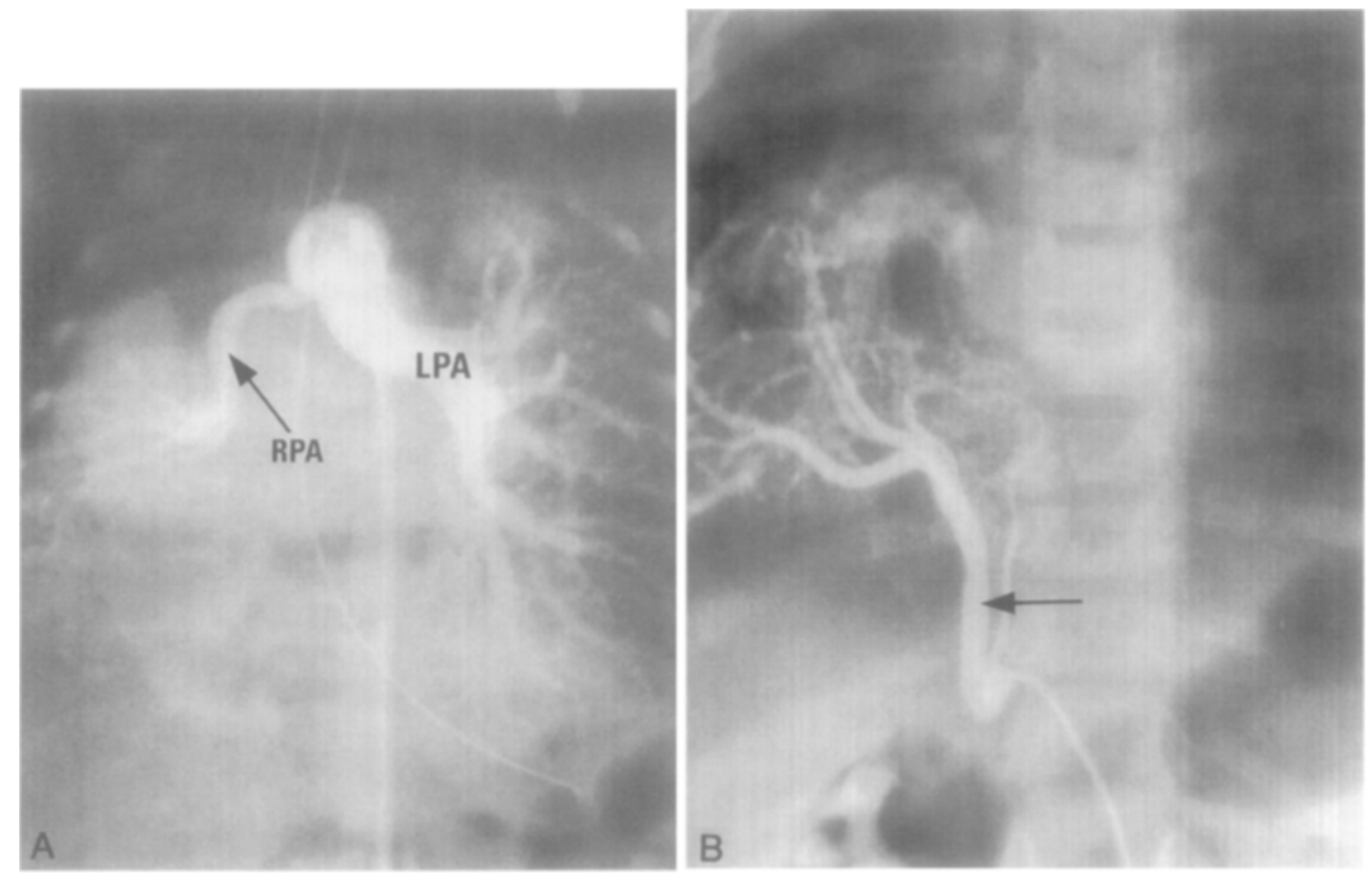

Fig. 2. A, Hypoplastic right pulmonary artery $(R P A)$. B, Systemic arterial supply to right lower lobe. $L P A$, Left pulmonary artery.

Table II. Preintervention hemodynamic parameters in 29 patients

\begin{tabular}{lccc}
\multicolumn{1}{c}{ Parameter } & $\begin{array}{c}\text { Infant } \\
(n=17)\end{array}$ & $\begin{array}{c}\text { Adult } \\
(n=12)\end{array}$ & $\begin{array}{c}\text { Total } \\
(n=29)\end{array}$ \\
\hline SVC (sat. \%) & 59 & 68 & 64 \\
IVC (sat. \%) & 80 & 84 & 83 \\
PA (sat. \%) & 76 & 84 & 80 \\
Aortic (sat. \%) & 94 & 97 & 95 \\
PAP (mean) & 30 & 21 & 26 \\
Aortic pressure (mean) & 67 & 99 & 83 \\
Ratio PAP/AoP (\%) & 44 & 22 & 33 \\
Qp/Qs & 2.1 & 2.6 & 2.4 \\
\hline
\end{tabular}

$S V C$, Superior vena cava; $I V C$, inferior vena cava; $P A$, pulmonary artery; $P A P$, pulmonary artery pressure; $A O P$, aortic pressure; $Q p / Q s$, pulmonary/ systemic flow ratio. Fourteen patients have a $Q \mathrm{p} / \mathrm{Qs}$ greater than $2: 1$, seven from each group.

${ }^{*} p<0.05$.

of right pulmonary artery stenoses $(n=1)$, open lung biopsy $(n=1)$, and closure of patent foramen ovale $(n=1)$.

Six members of the infantile group underwent intracardiac repair for anomalous pulmonary venous drainage at a median age of 2.4 years (range 0.5 to 5.8 years). They are discussed in the Surgical repair section.

Adult group. The syndrome was detected after the first year of life in 13 patients. The median age at presentation was 10.5 years (mean 18.5 years, range 2.5 to 70 years). There were six male and seven female patients. Symptoms were fatigue in two, dyspnea in three, and chest infections
Table III. Presentation and outcome of both groups

\begin{tabular}{lcc}
\hline & Infantile & Adult \\
\hline No. & 19 & 13 \\
Symptoms & 19 & 9 \\
Associated lesions (total) & 17 & 12 \\
Clinical outcome & & \\
Nonrepait & 13 & 2 \\
$\quad$ Deaths & 5 & 0 \\
$\quad$ Perfusion scan of right lung (\%) & 25 & N/A \\
Surgical repair & 6 & 11 \\
Postrepair pulmonary vein stenosis & 6 & 2 \\
CNS complications & 2 & 0 \\
Perfusion scan of right lung $(\%)$ & 19 & 27 \\
\hline
\end{tabular}

in four patients. Four patients were free of symptoms. Pulmonary hypertension was present in one patient only.

One patient underwent ligation of arterial collaterals and another had insertion of a permanent pacemaker. Eleven patients underwent intracardiac repair of anomalous pulmonary venous drainage at a median age of 11.3 years (mean 20 years, range 3.6 to 70.2 years) and are discussed in the Surgical repair section.

Surgical repair. Seventeen patients underwent intracardiac repair, six in the infantile group and 11 in the adult group. All intracardiac repairs were done with the patient supported by extracorporeal circulation with a mean bypass time of 94 minutes (range 42 to 195 minutes). Moderate systemic hypothermia was used in nine patients 
Table IV. Intervention other than repair in six patients

\begin{tabular}{lcc}
\multicolumn{1}{c}{ Procedure } & Infantile & Adult \\
\hline Occlusion of arterial supply & 5 & 1 \\
Coarctation repair & $1^{\dagger}$ & \\
Pneumonectomy & 1 & \\
PDA ligation & $1^{\dagger}$ & \\
Pulmonary vein & 1 & \\
RPA dilatation & 1 & \\
Closure of PFO & $1^{\dagger}$ & \\
Lung biopsy & $1^{\dagger}$ & \\
Pacemaker & 0 & 1 \\
Total & $1^{\dagger}$ & 2 \\
No intervention & $7^{\dagger}$ & 2
\end{tabular}

$\overline{P D A}$, Patent ductus arteriosus; $R P A$, right pulmonary artery; $P F O$, patent foramen ovale.

$\dagger$ Indicates death.

and deep hypothermic circulatory arrest was used in eight (six in the infantile group and two in the adult group) (mean 56 minutes, range 25 to 89 minutes). Myocardial protection included cold cardioplegia. Mean ischemic time was 62 minutes (range 24 to 130 minutes).

The intracardiac repair consisted of baffling the anomalous venous channel through the right atrium and into the left atrium through an atrial septal defect. The atrial defect was congenital in 12 patients and was created surgically in the five patients with an intact septum. The baffle was an autologous pericardial patch $(n=14)$ or a polytetrafluoroethylene patch $(n=3)$. Concomitant procedures were performed in 10 patients as follows: ligation of arterial collateral(s) in three (one of these patients required repair of aortic valve and IVC patch); patch enlargement of the IVC in four; and coarctation repair, ligation of the left superior vena cava, and pulmonary arterioplasty in one patient each.

All but one patient undergoing baffle repair had a subsequent follow-up study by echocardiogram $(n=16)$. Observations from this study led to cardiac catheterization in 10. Quantitative perfusion scans were performed in 15 of the patients who were treated surgically and in five of those who were not. The functional status of the patients was assessed and documented at follow-up. The surgically treated patients have been followed up for a mean of 8.9 years (range 1.6 to 17 years) after repair. Those not treated surgically have been followed up for a mean period of 5.7 years (range 0.7 to 18 years).

\section{Results}

Infantile group. Patients who were younger than 1 year old were more likely to have symptoms, had more associated lesions, had less favorable hemodynamics, and had a poorer clinical outcome than the patients who were older when the syndrome was identified (see Table IV). Seven of the infantile group have not required intervention. One of these infants died. Six infants had an intervention other than repair. Occlusion of arterial collaterals was undertaken in five infants (four by catheter technique and one by ligation). Three of these infants did not survive. All had associated procedures: ligation of a patent ductus arteriosus in one, lung biopsy in one (this showed significant medial hypertrophy, histologic Heath-Edwards grade 1, morphometric grade C), and repair of a patent foramen ovale in the third. The third infant had a left-to-right shunt of only 1.4:1 and died 2 months after atrial septal defect closure without repair of the anomalous vein.

One of the infants who had catheter occlusion of a collateral artery also had balloon dilatation and stenting of a stenotic right pulmonary vein. Although the child was clinically well, the pulmonary vein became occluded. One infant underwent coarctation repair and subsequent pneumonectomy and did not survive.

In total, there were five deaths among the 19 infants (26\%); all of these infants had pulmonary hypertension and died between the ages of 10 days and 7 months.

Six infants underwent baffle repair of the anomalous vein, the results of which are discussed in the following section.

Adult group. Eleven of the 13 patients in the adult group underwent intracardiac repair. Two patients have not undergone repair of the anomalous pulmonary vein. In each there is dual drainage to the IVC and to the left atrium. Their pulmonary blood flow is only 1.2 and 1.4 times systemic flow. One of these children, a 6 year old girl, underwent ligation of collaterals, and a pneumonectomy was considered but not done. Eleven years later, she is well although being treated for asthma. The other child was 15 years old when first evaluated, had no intervention, and has been well during 3 years of follow-up.

Surgical repair. All 17 patients have remained well during follow-up. However two required reoperation for severe obstruction in the intraatrial baffle.

An infant underwent coarctation and baffle repair at the age of 6 months. Her preoperative Qp/Qs was 3.7:1 with moderate pulmonary hypertension (50/20 $\mathrm{mm} \mathrm{Hg}$ ). Postoperative investigation demonstrated slow stenotic flow through the baffle, and revision was performed at age 2.7 years. At reoperation the site of obstruction was the junction of the anomalous vein with the IVC. A pericardial patch was inserted in the anomalous vein and in the IVC. Although she is clinically well at age 5 years, flow to 


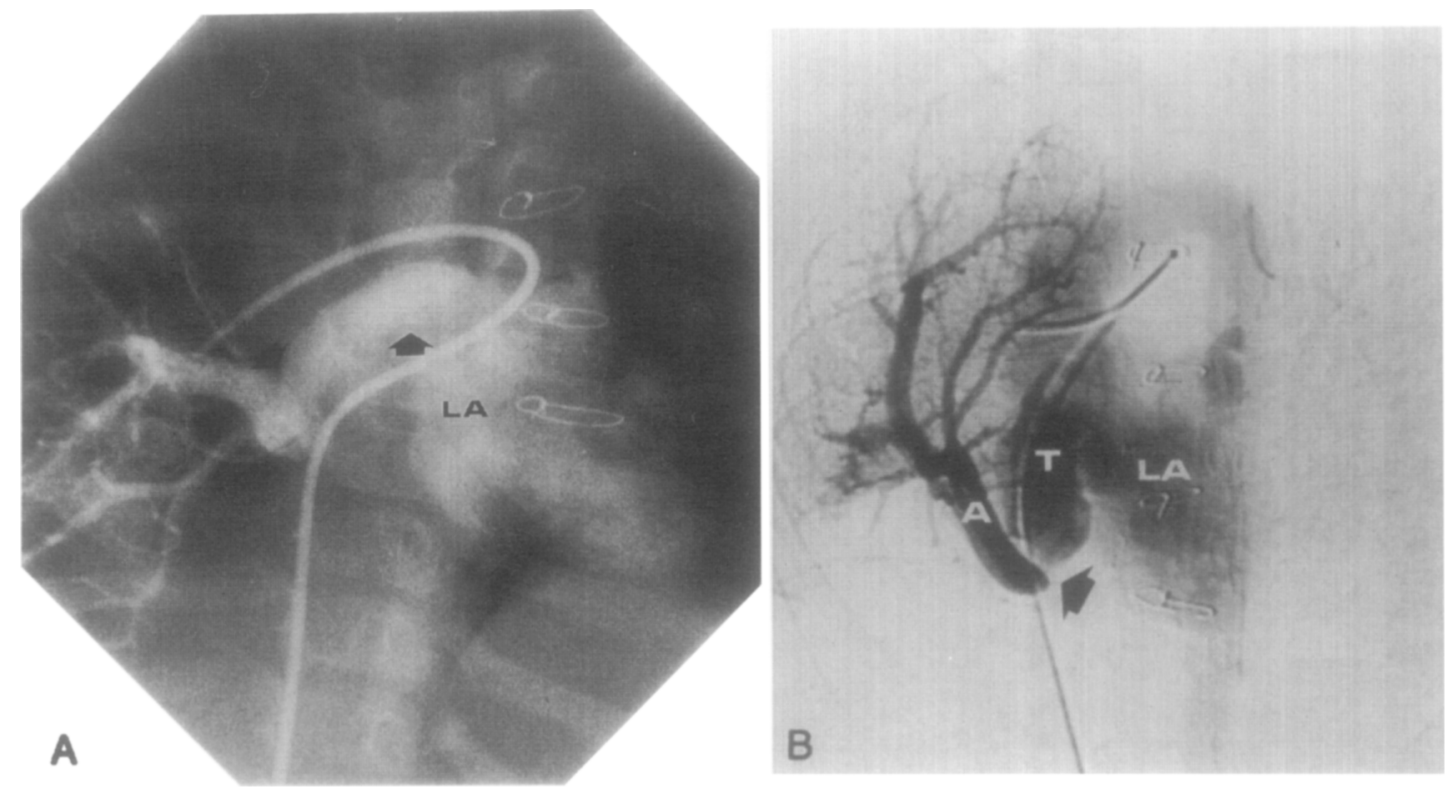

Fig. 3. A, Venous phase of a pulmonary angiogram showing a patent baffle repair through an atrial septal defect to the left atrium $(L A)$. B, Digital subtraction angiogram showing the anomalous vein $(A)$ and the tunnel $(T)$ to the left atrium $(L A)$. Note the site of stenosis (black arrow) at the junction of the anomalous vein with the baffle.

the right lung continues to be limited; on perfusion scan it is $27 \%$ compared with $73 \%$ to the left lung.

The other child who underwent reoperation had the initial operation at age 11 years and revision of the baffle 2 months later. Preoperative Qp/Qs was 2.8:1 with normal pulmonary artery pressure and an intact atrial septum. At reoperation the intraatrial baffle was completely occluded with thrombus. The atrial septum was closed and the vein was left draining into the right atrium. Despite an uneventful course in the 4 years since revision, his lung scan shows essentially no flow to the right lung.

One patient required permanent pacemaker implantation for sick sinus syndrome late after repair.

During follow-up 10 patients underwent cardiac catheterization. They had an average pulmonary artery pressure of $28 / 12 \mathrm{~mm} \mathrm{Hg}$, with an average mean of $18 \mathrm{~mm} \mathrm{Hg}$. There was no evidence of residual shunt, and the cardiac output was within normal limits. Postoperative pulmonary venous stenosis has been documented in a total of eight patients (47\%) (Fig. 3, $A$ and $B$ ).

Postoperative stenosis occurred in all six of the infantile group and in two of the 11 older patients. The site of obstruction or stenosis was at the junction of the anomalous vein to the IVC in six, in the tunnel in one and in the anomalous vein just before emerging from pulmonary parenchyma in the other.

Perfusion scan data for 15 patients who underwent repair and five others who did not undergo repair are presented in Table $\mathrm{V}$. The mean flow to the right lung was $24 \%$. In only three patients, all in the adult group, was the perfusion within normal limits.

\section{Discussion}

The scimitar syndrome consists of more than anomalous drainage of the right lung to the right side of the heart at the diaphragm. There are also important anomalies of the right lung, including hypoplasia and bronchial abnormalities, pulmonary artery and vein hypoplasia and stenosis, and collateral arterial supply to the right lower lobe from the descending aorta. There may also be important associated cardiac and noncardiac lesions.

Dupuis and associates ${ }^{6,7}$ collected data from 22 university centers and demonstrated important differences in patients whose diagnosis was made in the first year of life, which they named the "infantile" group, compared with those whose diagnosis was made after age 1 year, the "adult" group. In the 122 adult patients, there were four deaths, all among the 
Table V. Percent flow to right lung in perfusion scans in 20 patients

\begin{tabular}{ccc}
\hline Pt. No. & After repair & No repair \\
\hline 1 & 11 & 21 \\
2 & & 35 \\
4 & 6 & \\
6 & & 37 \\
7 & 27 & 0 \\
8 & 31 & \\
9 & 17 & \\
15 & & 30 \\
16 & 22 & \\
17 & 24 & \\
19 & 5 & \\
20 & 59 & \\
21 & 35 & \\
22 & 0 & \\
25 & 21 & \\
26 & 51 & \\
27 & 0 & 27 \\
29 & 51 & \\
30 & 24 & \\
32 & & \\
Mean $(\%)$ & & \\
Infantile (\%) & & \\
Adult (\%) & & \\
\hline
\end{tabular}

Patients 1 to 19 are in the infantile group.

37 patients who underwent repair. In contrast, there were 16 deaths among the 25 patients in the infantile group. Ligation of a patent ductus in five infants in Dupuis series was universally fatal. Conversely, ligation of the arterial collaterals was well tolerated in five of six infants and seemed to be beneficial. Pulmonary hypertension is frequent in this subset of patients, and this is attributed to multiple factors ${ }^{2,6}$ : (1) stenosis of the anomalous pulmonary vein; (2) the presence of systemic arterial supply to the right lung; (3) reduction of the pulmonary vascular bed on the right side; and (4) increased pulmonary blood flow resulting from the anomalous drainage or from the presence of an associated intracardiac defect. In our series $57 \%$ of the infantile group had pulmonary hypertension, one infant did not survive ductus ligation after catheter occlusion of collaterals, and two other deaths occurred among the five infants with occlusion of collaterals. Neill and associates ${ }^{2}$ recommended a two-staged approach to the infant-initial ligation of arterial collaterals followed by later repair of the anomalous vein. ${ }^{2}$ Torres and Dietl, ${ }^{8}$ in a study of 14 patients, also stressed the important differences between infants and older patients. In a previous publication from our institution, Gao and colleagues ${ }^{9}$ reported that 12 of 13 infants had pulmonary hypertension that was due to multiple causes including collateral arterial supply, pulmonary vein stenosis, pulmonary hypoplasia, and associated cardiac lesions.

In Dupuis' collected series of the adult form of the scimitar syndrome, ${ }^{7}$ thrombosis of the pulmonary venous baffle occurred in 17 of the 37 adults (46\%) who underwent repair and spontaneously in two with the adult form who did not undergo repair. The overall incidence of pulmonary venous obstruction in our series was $22 \%(7 / 32)$ at initial assessment and $47 \%(8 / 17)$ after repair. It was less common among patients with the adult form, two of 11 $(18 \%)$, and was universal among the six patients with the infantile form. In Dupuis' series, ${ }^{6}$ although 15 of the 25 infants had an operation, with six survivors, none had repair of the anomalous vein. Schramel and coworkers ${ }^{10}$ reported thrombosis in both patients in whom the vein was reimplanted into the left atrium.

Various techniques for intracardiac repair have been recommended: (1) direct implantation of the anomalous vein to the left atrium, ${ }^{11,12}$ (2) an intracardiac patch to baffle flow from the anomalous vein to the left atrium through an atrial septal defect (as was done in our series), either with the use of a polytetrafluoroethylene patch as described by Zubiate and $\mathrm{Kay}^{13}$ or with the use of right atrial wall as a patch, ${ }^{14}$ and (3) division and reimplantation of the anomalous vein into the right atrium and a baffle to redirect flow to the left atrium. ${ }^{8,15}$ The latter technique, used by Torres and Dietl ${ }^{8}$ in eight older patients, yielded good results with no evidence of pulmonary venous obstruction in the four patients studied after repair. Flow to the affected lung may be relatively small and the geometry of the anomalous vein and it's junction with the IVC makes the technical aspect of the repair critical.

In our study, the lung perfusion scan data give us important insight into the outcome of these patients. The best results are achieved in the adult form of scimitar syndrome; however, only three of 11 patients have normal right lung flow and two have venous obstruction. Although a better technical operative repair may improve these results, many patients with scimitar syndrome have very little flow through the anomalous vein, as evidenced by the perfusion data from the five patients who did not undergo repair; whose mean flow is $25 \%$. Reduced perfusion of the right lung may be due to a hypoplastic lung, to a small right pulmonary artery, or to pulmonary vein obstruction. Instinctively, surgeons 
like to correct the shunt through the anomalous vein. This decision to repair should be substantiated by quantifying this shunt as distinct from the other sources of shunts. Subsequently, repair should be undertaken only if the shunt is large (i.e., Qp/Qs $>$ 2:1). Preoperative calculation of anomalous pulmonary flow may be confounded by the presence of collateral arterial supply and by the presence of an atrial septal defect. Haworth, Sauer, and Buhlmeyer ${ }^{16}$ demonstrated that systemic arteries may connect with intrapulmonary arteries to distribute blood to a dilated capillary bed before reaching the pulmonary veins. Measuring the intracardiac shunt with the pulmonary artery open or occluded by a balloon catheter will allow calculation of the shunt from the anomalous vein independent of the other sources.

Pulmonary sequestration is a frequent occurrence in scimitar syndrome, as illustrated by the $43 \%$ incidence in our series. Bronchial sequestration is rarely present, although bronchial malformation is reported. ${ }^{4,10}$ There is an association between vascular sequestration and pulmonary hypoplasia, and probably congenital pulmonary vein stenosis. In addition, horseshoe lung was present in three patients in our series. This anomaly is closely associated with the scimitar syndrome. Freedom, Burrows, and Moes ${ }^{17}$ and Dupuis and coworkers ${ }^{18}$ reported on 11 new cases, nine of which were associated with scimitar syndrome. In a "horseshoe" lung, both lungs are fused at the posteroinferior segments behind the heart and in front of the esophagus. Usually the right pulmonary artery and right bronchi cross the midline to supply part of the left lung.

Surgical and percutaneous intervention for pulmonary venous obstruction has been reported, with poor results. ${ }^{19-21}$ Dickinson and colleagues ${ }^{22}$ reported improvement of symptoms with ligation or transcutaneous occlusion of the collateral arterial supply. There are no reports of lung ischemia after occlusion of the arterial collaterals. Bronchiectasis is rare in this form of vascular sequestration. Pulmonary resection has been reported by some authors ${ }^{23,24}$ to improve respiratory symptoms. Alivizatos and coworkers ${ }^{25}$ recommended a two-stage approach: the first stage is resection of the sequestered lung, and then repair of the pulmonary vein is carried out later in childhood. However, lung resection should be discouraged unless the lung is irreversibly damaged, because of the long-term effects of producing scoliosis in children or of producing chronic respiratory deficiency ${ }^{6}$ and because growth of the hypoplastic lung may occur as late as 7 to 10 years of age. ${ }^{7}$

\section{Conclusion}

Scimitar syndrome is a complex lesion in which the associated anomalies of the lung and heart are important determinants of management and prognosis. The infantile form is particularly complex and repair of the anomalous pulmonary vein has not been successful. Careful preoperative assessment should determine the importance of flow through the anomalous vein as distinct from left-to-right shunting through an associated cardiac defect, usually at the atrial level, and it should determine the importance of arterial collateral flow from the thoracic aorta to the lung. In the older patient, baffle repair is indicated in the presence of a substantial shunt (i.e., $>2: 1$ ), with the recognition that pulmonary venous and IVC stenosis is an inherent risk.

\section{REFERENCES}

1. Cooper G. Case of malformation of the thoracic viscera consisting of imperfect development of the right lung and transposition of the heart. London Med Gaz 1836;18:600-1.

2. Neill CA, Ferenca C, Sabiston DC, Sheldon $H$. The familial occurrence of hypoplastic right lung with systemic arterial supply and venous drainage, "scimitar syndrome." Johns Hopkins Med Bull 1960;107:1-21.

3. Kirklin JW, Ellis FH Jr, Wood WH. Treatment of anomalous pulmonary venous connection in association with interatrial communications. Surgery 1956;39:389-98.

4. Mathy J, Galey JJ, Logeais $Y$, et al. Anomalous pulmonary venous return into inferior vena cava and associated bronchovascular anomalies (the scimitar syndrome): report of three cases and review of the literature. Thorax 1968;23:398407.

5. Snellen HA, van Ingen HC, Hoefsmit ChM. Patterns of anomalous pulmonary venous drainage. Circulation 1968;38: 45-63.

6. Dupuis C, Charaf LAC, Breviere G, Abou P. "Infantile" form of the scimitar syndrome with pulmonary hypertension. Am J Cardiol 1993;71:1326-30.

7. Dupuis C, Charaf LAC, Breviere GM, Avoe P, Remy-Jardin M, Helmius G. The "adult" form of the scimitar syndrome. Am J Cardiol 1992;70:502-7.

8. Torres AR, Dietl CA. Surgical management of the scimitar syndrome: an age-dependent spectrum. Cardiovasc Surg 1993;1:432-8.

9. Gao YA, Burrows PE, Berison LN, Rabinovitch M, Freedom RM. Scimitat syndrome in infancy. J Am Coll Cardiol 1993;22:873-82.

10. Schramel FMNH, Westermann CJJ, Knaepen PJ, van den Bosch JMM. The scimitar syndrome: clinical spectrum and surgical treatment. Eur Respir J 1995;8:196-201.

11. Tornvall SS, Jackson KH, Alvayay JC, Varagas AC, Koch W, Zarate E. Anomalous drainage of the pulmonary veins into the inferior vena cava. J Thorac Cardiovasc Surg 1961;42: 413-7. 
12. Honey M. Anomalous pulmonary venous drainage of the right lung to inferior vena cava (scimitar syndrome): clinical spectrum in older patients and role of surgery. Q J Med 1977;46:463-83.

13. Zubiate P, Kay JH. Surgical correction of anomalous pulmonary venous connection. Ann Surg 1962;156:234-50.

14. Puig-Massana M, Murtra M, Revuelta JM. A new technique in the correction of partial anomalous pulmonary venous drainage. J Thorac Cardiovasc Surg 1972;64:108-13.

15. Shumacker HB, Judd D. Partial anomalous pulmonary venous return with reference to drainage into the inferior vena cava and to an intact septum. J Cardiovasc Surg (Torino) 1964;5:271-8.

16. Haworth SG, Sauer U, Buhlmeyer K. Pulmonary hypertension in scimitar syndrome in infancy. Br Heart J 1983;50: $182-9$.

17. Freedom RM, Burrows PE, Moes CA. "Horseshoe" lung: report of five new cases. AJR Am J Roentgenol 1986;146: 211-5.

18. Dupuis C, Remy J, Remy-Jardin M, Coulomb M, Breviere GM, Ben Laden S. The "horseshoe" lung: six new cases. Pediatr Pulmonol 1994;17:124-30.

19. Driscoll DJ, Hesslein PS, Mullins CE. Congenital stenosis of pulmonary veins: clinical spectrum and unsuccessful treatment by balloon dilatation. Am J Cardiol 1982;49:1767-72.

20. Coles JG, Yemets I, Najm HK, et al. Experience with repair of congenital heart defects using adjunctive endovascular devices. J Thorac Cardiovase Surg 1995;110:1513-20.

21. van de Wal HJCM, Goodman MJ, Harinck E, Lacquet LK, van Oort A. Pulmonary venous obstruction following correction for total anomalous pulmonary venous drainage: a challenge. 1992;6:545-9.

22. Dickinson DF, Gallaway RM, Massey R, Sankey R, Arnold R. Scimitar syndrome in infancy. Br Heart J 1982;47:468-72.

23. Thibault C, Perrault LP, Delisle G, Cartiet PC, Cloutier A, Houde $C$, et al. Lobectomy in the treatment of the scimitar syndrome. Ann Thorac Surg 1995;59:220-1.

24. Woody JN, Graham TP Jr, Bradford WD, Sabiston DC Jr, Canent RV Jr. Hypoplastic right lung with systemic blood supply and anomalous pulmonary venous drainage: reversal of pulmonary hypertension with surgical management in infancy. Am Heart J 1972;21:107-11.

25. Aliviziatos P, Cheatle T, de Leval M, Stark J. Pulmonary sequestration complicated by anomalies of pulmonary venous return. J Pediatr Surg 1985;20:76-9.

\section{Discussion}

Dr. Thomas L. Spray (Philadelphia, Pa.). I wish to thank the authors for bringing to our attention again the suboptimal results of operative intervention for patients with scimitar syndrome, even with the current state of our abilities to correct congenital heart defects. When considering discussion of this paper, I consulted one of my associates in Philadelphia, Dr. Bill Gaynor, who had looked up a series of all of the reported cases of scimitar syndrome in the English literature (136 references and 500 patients). He found 277 patients who had sufficient data to permit some kind of follow-up analysis.

According to the literature review, the results are abysmal in the infantile group, whether the patients are treated either medically or surgically. In fact, the same could be said for patients who have either medical treat- ment or surgical therapy in the adult group. They do not have very good long-term outcomes. One could question whether these patients should even come to surgical therapy.

Comparing the medical with the surgical treatment in the infant group in your series, I thought it was interesting that about $40 \%$ of the medically treated patients died and that although none of the infants who had a baffle type of repair died, yet all had pulmonary venous obstruction. This is an interesting phenomenon. It could be argued that an attempted repair in these patients might result in a functional pneumonectomy, but with the lung acting as a space-occupying but viable mass, therefore avoiding some of the complications of pneumonectomy in infants. Pneumonectomy is one approach that has been advocated for these types of patients with hypoplasia of the right lung.

Other series have shown that pulmonary hypertension is very prevalent in the infant group. Although you noted that the infant group did have a higher pulmonary pressure, it was still not at systemic levels. I wonder what the causes of death in the medically treated patients were. Did any of these patients die of pulmonary hypertension and the complications? In addition, did any of the surgically treated patients in the infant group have progressive pulmonary hypertension that may become sufficiently severe to require therapy in the future? I wonder whether some of these children will become similar to patients with congenital pulmonary vein stenosis, who have a very poor prognosis. One might consider lung transplantation as an option for some of these patients in the future.

Although the prevalence of pulmonary venous stenosis in the repair group in your series suggests that simple baffle repair is a poor choice in children with this condition, many other options for repair have been described. Most of the stenoses in your patient series, even in the older group, occurred at the pulmonary vein-IVC junction. When a baffle is created vertically, the right-angle connection of the pulmonary vein to the IVC almost invariably creates a junctional stenosis at that site. Many other options have been described, including displacement of the pulmonary vein to the right atrium and then baffling across the atrial septum. We have used a technique of cutting up into the right atrium along the venoatrial junction to bring the vein up into the right atrium, taking the angle away from the anastomosis and therefore decreasing the risk of junctional stenosis at that site. Would you consider other options of surgical repair in the future for older patients with this condition?

One of the problems with evaluating perfusion scans (and I noticed that none of your patients had a preoperative and postoperative perfusion scan) is that we really do not know the magnitude of flow to the scimitar lung in many of these patients. Although scimitar syndrome has a very wide spectrum of anatomic classifications, I noticed that only half of your patients had what you described as hypoplasia of the lung. Did the other patients have perfusion scans of the lung before repair?

Dr. Najm. Thank you, Dr. Spray, for your kind remarks.

The decision to attempt surgical repair in patients with scimitar syndrome is based on the shunt fraction. The mean shunt fraction in the medically treated patients is 
1.6:1 compared with $2.1: 1$ in the surgically treated patients. Granted, this is the total shunt; nevertheless, it gives us an idea that the right lung is hypoplastic, with very little blood flowing through it, and consequently the shunt is small. Baffling this blood back into the left atrium may not contribute to improvement in symptoms. For this reason it was elected not to attempt repair in this group of patients.

Five of 19 in the infantile group died, and all of these infants had pulmonary hypertension. Age at death was 10 days to 7 months. This subgroup of patients had complex congenital anomalies including small left ventricles, which precluded repair.

Regarding the type of repair, we elected to baffle the drainage from the anomalous vein through the right atrium to the atrial septal defect. Other techniques such a reimplantation of the anomalous vein into the left atrium have been reported, but anastomotic stenosis is prevalent. I agree with you that the angle created between the anomalous vein and the intracardiac tunnel may cause stenosis. A compromise technique reported by Honey and subsequently by Torres, wherein the anomalous vein is transplanted into the right atrial free wall and then a tunnel created to the atrial defect, may provide better results.

If we take a critical look at the reported cases of scimitar syndrome in the literature, we find that most of the patients have the adult form of the disease. All series reported before 1992 classification indicated the age at repair rather than the age at presentation, which is the basis of this classification, and it is difficult to discern whether these patients belong to the infantile group rather than the adult. Thus comparison between results in different centers is not valid without subgrouping the patients. In most reported series, the results of repair in the adult form are satisfactory, whatever the technique used. In contrast, repair is not often attempted in the infantile group, and the results are poor with current techniques.

Your suggestion of lung transplantation for the infantile group is very interesting and might be the treatment of choice.

None of our patients had both preoperative and postoperative perfusion scans, but as a result of the review we believe scanning should be an integral part of the preoperative evaluation. We have a group of five patients who did not undergo repair, and perfusion scans were abnormal in all. Although this group is not a true control, nevertheless their mean perfusion of the right lung is $25 \%$, almost identical to the mean postrepair perfusion in the surgically treated patients, only three of whom had normal perfusion.

\section{Bound volumes available to subscribers}

Bound volumes of The Journal of Thoracic and Cardiovascular Surgery are available to subscribers (only) for the 1996 issues from the Publisher, at a cost of $\$ 100.50$ for domestic, $\$ 128.94$ for Canadian, and $\$ 120.50$ for international subscribers for Vol, 111 (January-June) and Vol. 112 (July-December). Shipping charges are included. Each bound volume contains a subject and author index and all advertising is removed. Copies are shipped within 60 days after publication of the last issue of the volume. The binding is durable buckram with the Journal name, volume number, and year stamped in gold on the spine. Payment must accompany all orders. Contact Mosby-Year Book, Inc., Subscription Services, 11830 Westline Industrial Drive, St. Louis, Missouri 63146-3318, USA; phone 800-453-4351 or 314-453-4351.

Subscriptions must be in force to qualify. Bound volumes are not available in place of a regular Journal subscription. 